\title{
Conversely Convergence Theorem of Fabry Gap
}

\author{
Naser Abbasi, Molood Gorji* \\ Department of Mathematics, Faculty of science, Lorestan University, Khoramabad, Islamic Republic of Iran
}

\section{Email address:}

naserAbbasi_persia@yahoo.com (N. Abbasi),molodgorji@yahoo.com (M. Gorji)

\section{To cite this article:}

Naser Abbasi, Molood Gorji. Conversely Convergence Theorem of Fabry Gap. Science Journal of Applied Mathematics and Statistics. Vol. 3, No. 4, 2015, pp. 177-183. doi: 10.11648/j.sjams.20150304.12

\begin{abstract}
Our previous paper conducted to prove a variation of the converse of Fabry Gap theorem concerning the location of singularities of Taylor-Dirichlet series, on the boundary of convergence. In the present paper, we prove conversely convergence theorem of Fabry Gap. This is another proof of Fabry Gap theorem. This prove may be of interest in itself.
\end{abstract}

Keywords: Dirichlet Series, Entire Functions, Fabry Gap Theorem

\section{Introduction}

The Fabry Gap theorem ([11]) states that if, $\Lambda=\left\{\lambda_{n}\right\}_{n=1}^{\infty}$ is a real positive sequence such that $\lambda_{n+1}-\lambda_{n} \geq c$ for $c>0$ and $\frac{n}{\lambda_{n}} \mapsto D \geq 0$ as $n \mapsto \infty$, then the Dirichlet series $f(z)=\sum_{n=1}^{\infty} c_{n} e^{-\lambda_{n} z}$ has at least one singularity in every interval of length exceeding $2 \pi D$ on the abscissa of convergence.

We assume that the reader is familiar with the theory of Entire Functions and the theory of Dirichlet series, as used in the books [2,6,9-12].

We note that other results concerning the location of singularities of Taylor-Dirichlet series have been derived by Blambert, Parvatham, and Berland (see [3-5]).

\section{Auxiliary Results and Notions}

In this section, we describe the definitions and also to express and prove the lemma, we need to prove the theorem.

Definition 2.1. We denote by $L(c, D)$ the class of all sequences $A=\left\{a_{n}\right\}_{n=1}^{\infty}$ with distinct complex terms $a_{n}$ diverging to infinity, $\left|a_{n}\right| \leq\left|a_{n+1}\right|$ satisfying the following conditions: (see also [1])

(1) There is a constant $c>0$ so that $\left|a_{n}-a_{m}\right| \geq c|n-m|$ for all $n \neq m$.

(2) $\lim _{n \rightarrow \infty} n /\left|a_{n}\right|=D \geq 0$.
(3) the $\sup \left|\arg a_{n}\right|<\pi / 2$.

Definition 2.2. Let the sequence $A \in L(c, D)$ and $\alpha, \beta$ real positive numbers so that $\alpha+\beta<1$.

We say that a sequence $B=\left\{b_{n}\right\}_{n=1}^{\infty}$ with real positive terms $b_{n}$, not necessarily in an increasing order, belongs to the class $A_{\alpha, \beta}$ if for all $n \in N$ we have

$$
b_{n} \in\left\{z \in C:\left|z-a_{n}\right| \leq a_{n}^{\alpha}\right\}
$$

and for all $m \neq n$ one of the following holds:

$$
\begin{aligned}
& \text { (i) } b_{m}=b_{n} \\
& \text { (ii) }\left|b_{m}-b_{n}\right| \geq \max \left\{e^{-a_{m}^{\beta}}, e^{-a_{n}^{\beta}}\right\} .
\end{aligned}
$$

One observes that $(i)$ allows for the sequence $B$ to have coinciding terms. Also note that (ii) allows for non-coinciding terms to come very close to each other. We may now rewrite $B$ in the form of a multiplicity sequence $\Lambda=\left\{\lambda_{n}, \mu_{n}\right\}_{n=1}^{\infty}$, by grouping together all those terms that have the same modulus, and ordering them so that $\lambda_{n}<\lambda_{n+1}$. We shall call this form of $B$ the $(\lambda, \mu)$ reordering (see also [1]). given the sequences $A \in L(c, D)$ and $B \in A_{\alpha, \beta}$ (see also [14]):

$$
S_{1}=\bigcup_{n=1}^{\infty} B\left( \pm a_{n}, \frac{e^{-a_{n}^{\beta}}}{3}\right)
$$




$$
S_{2}=\bigcup_{n=1}^{\infty} B\left( \pm b_{n}, \frac{e^{-a_{n}^{\beta}}}{3}\right)
$$

where as usual

$$
B\left(z_{0}, r\right)=\left\{z:\left|z-z_{0}\right|<r\right\}
$$

Observe that the disks in $S_{2}$ is not necessarily disjoint, since for fixed $n$ we might have $b_{n}=b_{m}$ for $m \neq n$.

We state now lemmas that were proved in [1] regarding multiplicity sequences. They shall be used later on.

Lemma 2.3. Let $A \in L(c, D)$ be a real positive sequence and let $B \in A_{\alpha, \beta}$ so that $B=\left\{b_{n}\right\}$ is real positive too, with $(\lambda, \mu)$ its reordering. Then the regions of convergence of the three series $f, f^{*}, f^{* *}$ as defined in

$$
f(z)=\sum_{n=1}^{\infty} p_{\mu_{n}}(z) e^{-\lambda_{n} z}
$$

where $p_{\mu_{n}}(z)=\sum_{j=0}^{\mu_{n}-1} c_{n_{j}} z^{j} \quad$ is a polynomial with $c_{n_{\mu_{n}-1}} \neq 0$, and

$$
f^{*}(z)=\sum_{n=1}^{\infty} A_{n} e^{-\lambda_{n} z}, \quad f^{* *}(z)=\sum_{n=1}^{\infty} A_{n} z^{\mu_{n}-1} e^{-\lambda_{n} z}
$$

are the same. For any point $z$ inside the open convex region, the three series converge absolutely. Similarly, if instead of a real sequence $A \in L(c, D)$ we have a complex sequence $A \in L(c, 0)$.

Lemma 2.4. There exist positive constants $\psi$ and $\chi$ so that for any $n$ one has

$$
m(n) \leq \psi\left|a_{n}\right|^{\alpha} \leq \chi\left|b_{n}\right|^{\alpha}
$$

Lemma 2.5. For any $n$ one has

$$
\mu_{n} \leq \chi\left|\lambda_{n}\right|^{\alpha}
$$

Lemma 2.6. Let $\left\{L_{n}\right\}_{n=1}^{\infty}$ be anyone of the following sequences: $\left\{\lambda_{n}\right\},\left\{\lambda_{n} / 3\right\},\left\{\lambda_{n} \sin \gamma\right\}$ or $\left\{\lambda_{n}^{\alpha+\eta}\right\}$ where $\eta>0$ so that $\alpha+\beta+\eta<1$. Then

$$
\left|\sum_{j=0}^{\mu_{n}-1} c_{n_{j}} \sum_{l=0}^{j} \frac{j !}{(j-l) !}\left(\frac{1}{L_{n}}\right)^{l+1}(b+i B)^{j-l}\right|<A_{n}|b+i B|^{\mu_{n}-1} .
$$

Theorem 2.7. (Phragmen-Lindelof.) Let $f(z)$ be analytic in the region between two straight lines making an angle $\pi / \alpha$ at the origin and on the lines themselves. Suppose that

$$
|f(z)| \leq M
$$

on the lines and that as $r \rightarrow \infty$

$$
f(z)=O\left(e^{r \beta}\right)
$$

where $\beta<\alpha$, uniformly in the angle. Then (5) holds throughout the region.

Theorem 2.8. Let $A=\left\{a_{n}\right\}_{n=1}^{\infty}$ be a complex sequence satisfying $A \in L(c, D)$ and $\arg a_{n} \rightarrow 0$ as $n \rightarrow \infty$. Let $B \in A_{\alpha, \beta}$ and let $(\lambda, \mu)$ be its reordering. Then the entire function

$$
G(z)=\prod_{n=1}^{\infty}\left(1-\frac{z^{2}}{\lambda_{n}^{2}}\right)^{\mu_{n}}
$$

satisfies the following for every $\epsilon>0$ as $r \rightarrow \infty$ :

$$
\left|G\left(r e^{i \theta}\right)\right|=O(\exp \{\pi r(D|\sin \theta|+\epsilon)\}),
$$

and whenever $r e^{i \theta} \notin S_{2}$

$$
\frac{1}{\left|G\left(r e^{i \theta}\right)\right|}=O(\exp \{\pi r(-D|\sin \theta|+\epsilon)\})
$$

Furthermore for every $\epsilon>0$ as $n \rightarrow \infty$ one has:

$$
\frac{\mu_{n} !}{\left|G^{\left[\mu_{n}\right]}\left(\lambda_{n}\right)\right|}=O\left(\exp \left\{\epsilon\left|\lambda_{n}\right|\right\}\right)
$$

Valiron [13] (p. 29) proved that if $B=\left\{\lambda_{n}, \mu_{n}\right\}_{n=1}^{\infty}$ is a multiplicity-sequence that satisfies the relations

$$
\lim _{n \rightarrow \infty} \frac{\ln n}{\lambda_{n}}=0, \quad \lim _{n \rightarrow \infty} \frac{\mu_{n}-1}{\lambda_{n}}=0,
$$

the regions of convergence of the Taylor-Dirichlet series

$$
f(z)=\sum_{n=1}^{\infty} p_{\mu_{n}}(z) e^{-\lambda_{n} z}
$$

and its two associate series

$$
f^{*}(z)=\sum_{n=1}^{\infty} A_{n} e^{-\lambda_{n} z}, \quad f^{* *}(z)=\sum_{n=1}^{\infty} A_{n} z^{\mu_{n}-1} e^{-\lambda_{n} z}
$$

wher $A_{n}=\max \left\{\left|c_{n_{j}}\right|: j=0,1,2, \ldots, \mu_{n}-1\right\}$, are the same. For any point $z$ inside the open convex region, the three series converge absolutely.

\section{Main Results}

Theorem 3.1. Let $A \in L(c, D)$ be a real positive sequence for $D>0$. Let $B \in A_{\alpha, \beta}$ so that $B=\left\{b_{n}\right\}$ is real positive too and let $(\lambda, \mu)$ be its reordering. Then any Taylor-Dirichlet series $f(z)$ as in (3), satisfying

$$
\limsup _{n \rightarrow \infty} \frac{\ln \left|c_{n_{\mu_{n}-1}}\right|}{\lambda_{n}}=\limsup _{n \rightarrow \infty} \frac{\ln A_{n}}{\lambda_{n}}
$$

has at most one singularity in every interval of length 
exceeding $2 \pi D$ on the abscissa of convergence.

Proof. We follow on the lines of the proof of Theorem XXIX in [11].

Let $f(z), f^{*}(z), f^{* *}(z)$ and $A_{n}$ as defined in (3), (11) and

$$
A_{n}=\max \left\{\left|c_{n_{j}}\right|: j=0,1,2, \ldots, \mu_{n}-1\right\}
$$

From Lemma 2.3, the regions of convergence of the three series are the same. Since the $\lambda_{n}$ are real positive numbers, we consider the non-trivial case, that is when the three series converge in identical half-planes of the form $\Re z>x_{0}, x_{0} \in R$.

With no loss of generality we assume that the abscissa of convergence (ordinary and absolute) is the line $x=0$. In other words the relation

$$
\limsup _{n \rightarrow \infty} \frac{\operatorname{Ln} A_{n}}{\lambda_{n}}=0
$$

holds. Thus, all three series converge absolutely and uniformly in any half-plane $x \geq \tau>0$. One also notes that from (12) we have

$$
\limsup _{n \rightarrow \infty} \frac{\operatorname{Ln}\left|c_{n_{\mu_{n-1}}}\right|}{\lambda_{n}}=0 .
$$

Suppose now that there exists an interval of length greater than $2 \pi D$ on the line $x=0$ on which $f(z)$ has two singularity $x_{1}, x_{2}$.

Then with no loss of generality we can also assume that this interval is $-B \leq y \leq B$ where $B>\pi D$. This implies the existence of some $a_{1}, a_{2}>0$ such that $\$ \mathrm{f}(\mathrm{z}) \$$ is analytic for $x_{1} \geq-a_{1}, x_{2} \geq-a_{2},\left|y_{1}\right| \leq B,\left|y_{2}\right| \leq B$. We put

$$
\begin{aligned}
& \gamma_{1}=\arctan \frac{a_{1}}{4 \pi D}, \\
& \gamma_{2}=\arctan \frac{a_{2}}{4 \pi D} .
\end{aligned}
$$

so that $0<\gamma_{1}, \gamma_{2}<\pi / 2$ and let

$$
\begin{aligned}
& b_{1}=\frac{(B-\pi D) \tan \gamma_{1}}{2}, \\
& b_{2}=\frac{(B-\pi D) \tan \gamma_{2}}{2} .
\end{aligned}
$$

The rest of the proof is broken into three steps. (to prove that $x_{1}, x_{2}$ can be obtained similarly.)

All three steps make use of the convergence of

$$
\sum_{n=1}^{\infty} A_{n}\left|b_{1}+i B\right|^{\mu_{n}-1}\left|e^{-\lambda_{n}\left(b_{1}+i B\right)}\right|,
$$

due to $f^{* *}\left(b_{1}+i B\right)$.

Step 1:

Since $f(z)$ is regular in the semi-strip $\left(x_{1} \geq-a_{1},\left|y_{1}\right| \leq B\right)$, then for all $w \in C$ so that $\mathfrak{R} w<0$, we define

$$
\begin{gathered}
H(R, w)=\int_{-a_{1}}^{-a_{1}+i B} f(z) e^{w z} d z+\int_{-a_{1}+i B}^{b_{1}+i B} f(z) e^{w z} d z \\
+\int_{b_{1}+i B}^{R+i B} f(z) e^{w z} d z+\int_{R+i B}^{R} f(z) e^{w z} d z
\end{gathered}
$$

where $R>b_{1}$ and the paths of the integrals are the segments joining the various points. Then we prove that $H(R, w)$ converges as $R \rightarrow \infty$, and if we denote this limit by $H(w)$, one has

$$
\begin{gathered}
H(w)=\int_{-a_{1}}^{-a_{1}+i B} f(z) e^{w z} d z+\int_{-a_{1}+i B}^{b_{1}+i B} f(z) e^{w z} d z \\
+\sum_{n=1}^{\infty} e^{\left(b_{1}+i B\right)\left(w-\lambda_{n}\right)} \sum_{j=0}^{\mu_{n}-1} c_{n_{j}} \sum_{l=0}^{j} \frac{(-1)^{l+1}\left(b_{1}+i B\right)^{j-l}}{\left(w-\lambda_{n}\right)^{l+1}} \frac{j !}{(j-l) !} .
\end{gathered}
$$

But now $H(w)$ is well defined for all $w \in C \backslash\left\{\lambda_{n}\right\}$. In fact it is analytic in $C \backslash\left\{\lambda_{n}\right\}$.

Next, we define $J(w)=H(w) G(w)$ where $G(w)$ is the entire even function defined in Theorem 2.8 Then $J(w)$ is an entire function in the complex plane.

Proof step 1:

Observe that the first two integrals in (21) are independent of $R$, thus we deal with the other two. We will prove that (22) holds as $R \rightarrow \infty$.

The absolute convergence of $f(z)$ in the interval $\left[b_{1}+i B, R+i B\right]$, justifies integrating it term by term to get the following:

$$
\begin{aligned}
& \int_{b_{1}+i B}^{R+i B} f(z) e^{w z} d z= \\
& -\sum_{n=1}^{\infty} e^{(R+i B)\left(w-\lambda_{n}\right)} \sum_{j=0}^{\mu_{n}-1} c_{n_{j}} \sum_{l=0}^{j} \frac{(-1)^{l+1}(R+i B)^{j-l}}{\left(w-\lambda_{n}\right)^{l+1}} \frac{j !}{(j-l) !} \\
& +\sum_{n=1}^{\infty} e^{\left(b_{1}+i B\right)\left(w-\lambda_{n}\right)} \sum_{j=0}^{\mu_{n}-1} c_{n_{j}} \sum_{l=0}^{j} \frac{(-1)^{l+1}\left(b_{1}+i B\right)^{j-l}}{\left(w-\lambda_{n}\right)^{l+1}} \frac{j !}{(j-l) !}
\end{aligned}
$$

Denote by $I(R, w)$ the infinite series which depends on $R$. We will show that $I(R, w) \rightarrow 0$ as $R \rightarrow \infty$.

Since $\Re w<0$ then $\left|w-\lambda_{n}\right|>\lambda_{n}$. It follows from Lemma 2.6 that

$$
\left|\sum_{j=0}^{\mu_{n}-1} c_{n_{j}} \sum_{l=0}^{j} \frac{(-1)^{l+1}(R+i B)^{j-l}}{\left(w-\lambda_{n}\right)^{l+1}} \frac{j !}{(j-l) !}\right| \leq A_{n}|R+i B|^{\mu_{n}-1} .
$$

Hence 


$$
\begin{aligned}
|I(R, w)| & \leq \sum_{n=1}^{\infty} A_{n}|R+i B|^{\mu_{n}-1}\left|e^{(R+i B)\left(w-\lambda_{n}\right)}\right| \\
& \leq e^{-B \mathfrak{S}_{w}} \sum_{n=1}^{\infty} A_{n}(2 R)^{\mu_{n}-1} e^{-R \lambda_{n}}
\end{aligned}
$$

On the other hand, since $\mu_{n} / \lambda_{n} \rightarrow 0$ one has $(2 R)^{\mu_{n}-1}<e^{\left(\lambda_{n} R\right) / 4}$ and therefore

$$
|I(R, w)|<e^{-B \mathfrak{S} w} \sum_{n=1}^{\infty} 2 A_{n} e^{-\left((3 R / 4) \lambda_{n}\right)} \leq e^{-B \mathfrak{W} w} e^{-(R / 4) \lambda_{1}} \sum_{n=1}^{\infty} A_{n} e^{-R\left(\lambda_{n} / 2\right)} .
$$

From relation (14) one gets that $\limsup _{n \rightarrow \infty}\left(\ln A_{n}\right) /\left(\lambda_{n} / 2\right)=0$, and this implies that the Dirichlet series $f^{* * *}(z)=\sum_{n=1}^{\infty} A_{n} e^{-\left(\lambda_{n} / 2\right) z}$ converges absolutely for any $z$ if $\mathfrak{R} z>0$. Thus, the series

$$
f^{* * *}(R)=\sum_{n=1}^{\infty} A_{n} e^{-\left(\lambda_{n} / 2\right) R}
$$

is defined for all $R>0$ and is a positive decreasing function. Therefore there exists some $M>0$ so that for all $R>1$ one has $f^{* * *}(R)<M$. Combining this with (23) shows that $I(R, w) \rightarrow 0$ as $R \rightarrow \infty$.

Similarly one deduces that

$$
\lim _{R \rightarrow \infty} \int_{R+i B}^{R} f(z) e^{w z} d z=0 .
$$

Therefore for all $w$ with $\mathfrak{R} w<0$ one has that the $\lim _{R \rightarrow \infty} H(R, w)$ exists. If we denote this by $H(w)$ then $H(w)$ takes the form as in (22).

Next we prove that $H(w)$ is well defined for all $w \in C \backslash\left\{\lambda_{n}\right\}$. In fact, we prove that $H(w)$ is analytic in $C \backslash\left\{\lambda_{n}\right\}$.

Note that the two integrals in (22) define analytic functions of $w$ in the whole complex plane. Thus, it remains to be proved that the infinite series converges uniformly on any compact subset $K \in C$ such that $K \cap\left\{\lambda_{n}\right\}_{n=1}^{\infty}=\varnothing$.

Consider such a compact $K$. Then there exists an $n_{0} \in N$ so that for all $w \in K$ one has $\left|w-\lambda_{n}\right| \geq \lambda_{n} / 2$ for all $n \geq n_{0}$. Let $q=\max \left\{\left|e^{\left(b_{1}+i B\right) w}\right|: w \in K\right\}$. For all $w \in K$ define

$$
I_{n_{0}}(w)=\sum_{n=n_{0}}^{\infty} e^{\left(b_{1}+i B\right)\left(w-\lambda_{n}\right)} \sum_{j=0}^{\mu_{n}-1} c_{n_{j}} \sum_{l=0}^{j} \frac{(-1)^{l+1}\left(b_{1}+i B\right)^{j-l}}{\left(w-\lambda_{n}\right)^{l+1}} \frac{j !}{(j-l) !} .
$$

Then for all $w \in K$, it follows from Lemma 2.6 and (20) that

$$
\left|I_{n_{0}}(w)\right| \leq q \sum_{n=n_{0}}^{\infty} A_{n}\left|b_{1}+i B\right|^{\mu_{n}-1}\left|e^{-\lambda_{n}\left(b_{1}+i B\right)}\right| \rightarrow 0, \quad n_{0} \rightarrow \infty .
$$

This implies uniform convergence on $K$.

Step 2:

We prove that for some $\delta_{1}>0$ the relation $\left|J\left(\rho e^{ \pm i \gamma_{1}}\right)\right|=O\left(e^{-\delta_{1} \rho \cos \gamma_{1}}\right)$ holds, thus $\left|e^{\delta_{1} w} J(w)\right|=O$ (1) for $\arg w= \pm \gamma_{1}$.

Proof step 2:

Let $\gamma_{1}$ be as in (16). Then

$$
\begin{aligned}
& H\left(\rho e^{i \gamma_{1}}\right)=\int_{-a_{1}}^{-a_{1}+i B} f(z) e^{z \rho e^{i \eta_{1}}} d z+\int_{-a_{1}+i B}^{b_{1}+i B} f(z) e^{z \rho e^{i \eta_{1}}} d z \\
& +\sum_{n=1}^{\infty} e^{\left(b_{1}+i B\right)\left(\rho e^{i n}-\lambda_{n}\right)} \sum_{j=1}^{\mu_{n}} c_{n_{j}} \sum_{l=0}^{\mu_{n}-1} \frac{(-1)^{l+1}\left(b_{1}+i B\right)^{j-l}}{\left(\rho e^{i \gamma_{1}}-\lambda_{n}\right)^{l+1}} \frac{j !}{(j-l) !} .
\end{aligned}
$$

Denote the infinite series by $T\left(\rho e^{i \gamma_{1}}\right)$ and note that $\left|\rho e^{i \gamma_{1}}-\lambda_{n}\right| \geq \lambda_{n} \sin \gamma_{1}$. Then from

Lemma 2.6 and (20), it follows that

$$
\left|T\left(\rho e^{i \gamma_{1}}\right)\right|=O\left(e^{b_{1} \rho \cos \gamma_{1}-B \rho \sin \gamma_{1}}\right) .
$$

Since $f(z)$ is bounded on the segments $\left[-a_{1},-a_{1}+i B\right]$ and $\left[-a_{1}+i B, b_{1}+i B\right]$, by standard calculations one gets

$$
\left|\int_{-a_{1}}^{-a_{1}+i B}+\int_{-a_{1}+i B}^{b_{1}+i B}\right|=O\left(e^{-a \rho \cos \gamma_{1}}+e^{b \rho \cos \gamma_{1}-B \rho \sin \gamma_{1}}\right) .
$$

Then by choosing the path of integration as the reflection in the real axis of that used in (21), we get that (25) and (26) hold for $\rho e^{-i \gamma_{1}}$ as well. Thus

$$
H\left(\rho e^{ \pm i \gamma_{1}}\right)=O\left(e^{-a_{1} \rho \cos \gamma_{1}}+e^{b_{1} \rho \cos \gamma_{1}-B \rho \sin \gamma_{1}}\right) .
$$

From the definition of $J(w)$ above, one deduces that

$$
J(w)=G(w) \int_{-a_{1}}^{-a_{1}+i B} f(z) e^{w z} d z+G(w) \int_{-a_{1}+i B}^{b_{1}+i B} f(z) e^{w z} d z+Q(w)
$$

where $Q(w)$ is the entire function defined as

$$
G(w) \sum_{n=1}^{\infty} e^{\left(b_{1}+i B\right)\left(w-\lambda_{n}\right)} \sum_{j=0}^{\mu_{n}-1} c_{n_{j}} \sum_{l=0}^{j} \frac{(-1)^{l+1}\left(b_{1}+i B\right)^{j-l}}{\left(w-\lambda_{n}\right)^{l+1}} \frac{j !}{(j-l) !} .
$$

Note that $Q(w)$ is also written as 


$$
\sum_{n=1}^{\infty} e^{\left(b_{1}+i B\right)\left(w-\lambda_{n}\right)} \sum_{j=0}^{\mu_{n}-1} c_{n_{j}} \sum_{l=0}^{j} \frac{(-1)^{\mu_{n}+l+1}\left(b_{1}+i B\right)^{j-l} j !}{(j-l) !}\left(\lambda_{n}-w\right)^{\mu_{n}-l-1} G_{n}(w)
$$

where

$$
G_{n}(w)=\frac{\left(\lambda_{n}+w\right)^{\mu_{n}}}{\lambda_{n}^{2 \mu_{n}}} \prod_{k \neq n}\left(1-\frac{w^{2}}{\lambda_{k}^{2}}\right)^{\mu_{k}}
$$

One observes that combining (7) and (27), gives for every $\epsilon>0$

$$
\left|J\left(\rho e^{ \pm i \gamma_{1}}\right)\right|=O\left(e^{-a_{1} \rho \cos \gamma_{1}+\pi \rho D \sin \gamma_{1}+\epsilon \rho}+e^{b_{1} \rho \cos \gamma_{1}+(\pi D-B) \rho \sin \gamma_{1}+\epsilon \rho}\right) .
$$

From (18) one also deduces that $B-\pi D=2 b \cot \gamma_{1}$, and since $\epsilon$ is arbitrarily small this yields

$$
\left|J\left(\rho e^{ \pm i \gamma_{1}}\right)\right|=O\left(e^{-\left(a_{1} / 2\right) \rho \cos \gamma_{1}+\pi \rho D \sin \gamma_{1}}+e^{-\left(b_{1} / 2\right) \rho \cos \gamma_{1}}\right) .
$$

Relation (16) implies that $\pi D \sin \gamma_{1}=1 / 4 a_{1} \cos \gamma_{1}$, thus for $\delta_{1}=1 / 4 \min \left(a_{1}, b_{1}\right)$ we have

$$
\left|J\left(\rho e^{ \pm i \gamma_{1}}\right)\right|=O\left(e^{-\delta_{1} \rho \cos \gamma_{1}}\right) .
$$

Therefore

$$
\left|e^{\delta_{1} w} J(w)\right|=O(1), \quad \arg w= \pm \gamma_{1} .
$$

Step 3:

We show that $e^{\delta_{1} w} J(w)$ is a function of exponential type bounded in the angle $|\arg w| \leq \gamma_{1}$. In particular, for real $w$ this implies that $J(w)=O\left(e^{-\delta_{1} w}\right)$, thus $J\left(\lambda_{m}\right)=O\left(e^{-\delta_{1} \lambda_{m}}\right)$. This eventually yields the relation $\left|c_{m_{\mu_{m-1}}}\right|=O\left(e^{-\left(\delta_{1} / 2\right) \lambda_{m}}\right)$ which contradicts relation (15). So, two points can be obtained in two different, but the relationship is bound (15) both should be equal to zero, then there is either a single point, and this completes the proof of the theorem.

Proof step 3:

We will show that (32) holds in the angle $|\mathfrak{I} w| \leq \gamma_{1}$. In order to do this, first we prove that $J(w)$ is an entire function of exponential type. From (28) observe that it suffices to work with the function $Q(w)$.

Consider some $\eta>0$ so that $\alpha+\beta+\eta<1$. For every $w \in C$ so that $w \notin S_{2}$ where $S_{2}$ is the system defined in (2), we partition the sequence $\left\{\lambda_{n}\right\}$ into two sets as follows:

$$
A(w)=\left\{\lambda_{n}:\left|w-\lambda_{n}\right|>\lambda_{n}^{\alpha+\eta}\right\}
$$

and

$$
B(w)=\left\{\lambda_{n}:\left|w-\lambda_{n}\right| \leq \lambda_{n}^{\alpha+\eta}\right\}
$$

Then we write

$$
Q(w)=Q_{A}(w)+Q_{B}(w)
$$

where $Q_{A}(w)$ is defined as

$$
Q_{A}(w)=G(w) \sum_{\lambda_{n} \in A(w)} e^{\left(b_{1}+i B\right)\left(w-\lambda_{n}\right)} \sum_{j=0}^{\mu_{n}-1} c_{n_{j}} \sum_{l=0}^{j} \frac{(-1)^{l+1}\left(b_{1}+i B\right)^{j-l}}{\left(w-\lambda_{n}\right)^{l+1}} \frac{j !}{(j-l) !} .
$$

Similarly one defines $Q_{B}(w)$.

Consider now $Q_{A}(w)$. We remark that in this case the condition $w \notin S_{2}$ plays no role. Note that from Lemma 2.6, we deduce for any $\lambda_{n} \in A(w)$ that

$$
\left|\sum_{j=0}^{\mu_{n}-1} c_{n_{j}} \sum_{l=0}^{j} \frac{(-1)^{l+1}\left(b_{1}+i B\right)^{j-l}}{\left(w-\lambda_{n}\right)^{l+1}} \frac{j !}{(j-l) !}\right| \leq A_{n}\left|b_{1}+i B\right|^{\mu_{n}-1}
$$

$$
\left|Q_{A}(w)\right| \leq|G(w)|\left|e^{\left(b_{1}+i B\right) w}\right| \sum_{\lambda_{n} \in A(w)} A_{n}\left|b_{1}+i B\right|^{\mu_{n}-1}\left|e^{-\lambda_{n}\left(b_{1}+i B\right)}\right|,
$$

and observe that the series is bounded above by the one in (20). This implies that $\left|Q_{A}(w)\right|=O\left(e^{\kappa|w|}\right)$ for some $\kappa>0$.

Next, we consider $Q_{B}(w)$. We can also write it as

Thus

$$
\sum_{\lambda_{n} \in B(w)} e^{\left(b_{1}+i B\right)\left(w-\lambda_{n}\right)}\left[\sum_{j=0}^{\mu_{n}-1} c_{n_{j}} \sum_{l=0}^{j} \frac{\left(b_{1}+i B\right)^{j-l} j !}{(-1)^{l+1}(j-l) !}\left(\lambda_{n}-w\right)^{\mu_{n}-l-1}\right] G_{n}(w),
$$

where $G_{n}(w)$ is defined in (31). Note that for any $\lambda_{n}$ there is a $b_{1_{j}}$ so that $\lambda_{n}=b_{1_{j}}$, thus $\mu_{n}=m(j)$ the pseudo-multiplicity of $b_{1_{j}}$. Since $w \notin S_{2}$, then one gets

$$
\left|G_{n}(w)\right|=\frac{|G(w)|}{\left|\lambda_{n}-w\right|^{\mu_{n}}}=\frac{|G(w)|}{\left|b_{1_{j}}-w\right|^{m(j)}} \leq|G(w)|\left(3 e^{\mid a_{1_{j}} j^{\beta}}\right)^{m(j)} .
$$


Fix some $>0$. Then from Lemma 2.4 we get

$$
\left(3 e^{\left|a_{1 j}\right|^{\beta}}\right)^{m(j)} \leq\left(3 e^{\left|a_{1 j}\right|^{\beta}}\right)^{\psi\left|a_{1 j}\right|^{\alpha}}=3 e^{\psi\left|a_{1 j}\right|^{\alpha+\beta}} \leq e^{\epsilon\left|a_{1 j}\right|},
$$

$$
\left|G_{n}(w)\right| \leq A^{\prime} e^{A^{\prime \prime}|w|}
$$

Next, observe that for any $\lambda_{n} \in B(w)$, we have

$$
\left|\lambda_{n}-w\right|^{\mu_{n}-l-1} \leq\left(\lambda_{n}^{\alpha+\eta}\right)^{\mu_{n}-l-1}
$$

with the last inequality valid since $\alpha+\beta<1$. One also observes that $\left|a_{1_{j}}\right| \leq 2\left|b_{1_{j}}\right|=2 \lambda_{n} \leq 4|w|$ since $\lambda_{n} \in B(w)$. Thus for all $\lambda_{n} \in B(w)$ one has $\left|G_{n}(w)\right| \leq|G(w)| e^{4 \epsilon|w|}$. This implies that there are constants $A^{\prime}>0$ and $A^{\prime \prime}>0$, so that for any $w \in C \backslash S_{2}$ and all $\lambda_{n} \in B(w)$ one has

$$
A^{\prime} e^{A^{\prime \prime}|w|} \sum_{\lambda_{n} \in B(w)}\left|e^{\left(b_{1}+i B\right)\left(w-\lambda_{n}\right)}\right|\left(\lambda_{n}^{\alpha+\eta}\right)^{\mu_{n}} \sum_{j=0}^{\mu_{n}-1}\left|c_{n_{j}}\right| \sum_{l=0}^{j} \frac{\left|b_{1}+i B\right|^{j-l} j !}{(j-l) !\left(\lambda_{n}^{\alpha+\eta}\right)^{l+1}}
$$

Then from Lemma 2.6 we get that

$$
\left|Q_{B}(w)\right| \leq A^{\prime} e^{A^{\prime \prime}|w|}\left|e^{\left(b_{1}+i B\right) w}\right| \sum_{\lambda_{n} \in B(w)}\left(\lambda_{n}^{\alpha+\eta}\right)^{\mu_{n}} A_{n}\left|b_{1}+i B\right|^{\mu_{n}-1}\left|e^{-\lambda_{n}\left(b_{1}+i B\right)}\right| .
$$

Note also that from Lemma 2.5 one gets

$$
\left(\lambda_{n}^{\alpha+\eta}\right)^{\mu_{n}} \leq\left(\lambda_{n}^{\alpha+\eta}\right)^{\chi \lambda_{n}^{\alpha}}=\lambda_{n}^{\chi(\alpha+\eta) \lambda_{n}^{\alpha}} \leq e^{\epsilon \lambda_{n}} \leq e^{2 \epsilon|w|}
$$

and combining this with (37) gives

$$
\left|Q_{B}(w)\right| \leq A^{\prime} e^{A^{\prime \prime}|w|}\left|e^{\left(b_{1}+i B\right) w}\right| e^{2 \epsilon|w|} \sum_{\lambda_{n} \in B(w)} A_{n}\left|b_{1}+i B\right|^{\mu_{n}-1}\left|e^{-\lambda_{n}\left(b_{1}+i B\right)}\right|,
$$

with the series bounded above by the one in (20). This implies that $\left|Q_{B}(w)\right|=O\left(e^{\sigma|w|}\right)$ for some $\sigma>0$, provided $w \notin S_{2}$.

Since $Q(w)=Q_{A}(w)+Q_{B}(w)$ it follows that $|Q(w)|=O\left(e^{v|w|}\right)$ for some $v>0$, provided $w \notin S_{2}$. But according to

$$
\bigcup_{m \in \Gamma_{n}} B\left(b_{m}, \frac{e^{-\left|a_{m}\right|^{\beta}}}{3}\right)=B\left(b_{n}, \frac{e^{-\mid a_{\left.l_{n}\right|^{\beta}}}}{3}\right), \quad l_{n}=\min \left\{m: m \in \Gamma_{n}\right\}
$$

$S_{2}$ is the union of non-overlapping disks whose radius tends to zero. Since $Q(w)$ is an entire function, its maximum value over any such closed disk is obtained on the boundary. All these imply that $|Q(w)|=O\left(e^{v|w|}\right)$ for all $w$. It then follows that $J(w)$ is an entire function of exponential type. Combining this result with relation (32) and a Phragmen-Lindelof theorem 2.7, it yields

$$
\left|e^{\delta_{1} w} J(w)\right|=O(1), \quad|\arg w| \leq \gamma_{1} .
$$

In particular, for real $w$ this implies that $J(w)=O\left(e^{-\delta_{1} w}\right)$, thus

$$
J\left(\lambda_{m}\right)=O\left(e^{-\delta_{1} \lambda_{m}}\right)
$$

Note also that from (28) and (29), one deduces that

$$
J\left(\lambda_{m}\right)=\frac{c_{m_{\mu_{m}-1}} 2^{\mu_{m}}\left(\mu_{m}-1\right) !}{\lambda_{m}^{\mu_{m}}} \prod_{k \neq m}\left(1-\frac{\lambda_{m}^{2}}{\lambda_{k}^{2}}\right)^{\mu_{k}}
$$

Then from

$$
\frac{G^{\left[\mu_{n}\right]}\left(\lambda_{n}\right)}{\mu_{n} !}=\frac{(-2)^{\mu_{n}}}{\lambda_{n}^{\mu_{n}}} \prod_{k \neq n}\left(1-\frac{\lambda_{n}^{2}}{\lambda_{k}^{2}}\right)^{\mu_{k}}
$$

we can write

$$
c_{m_{\mu_{m}-1}}=J\left(\lambda_{m}\right)\left[\frac{\mu_{m} !}{G^{\left[\mu_{m}\right]}\left(\lambda_{m}\right)}\right]\left[\frac{1}{(-1)^{\mu_{m}}\left(\mu_{m}-1\right) !}\right] .
$$

If we now apply (9) and (40) - (43), it yields for every $\epsilon>0$

$$
\left|c_{m_{\mu_{m}-1}}\right|=O\left(e^{\left(-\delta_{1}+\epsilon\right) \lambda_{m}}\right) .
$$


Since is arbitrary we get that

$$
\begin{aligned}
& \left|c_{m_{\mu_{m-1}}}\right|=O\left(e^{\left(-\delta_{1} / 2\right) \lambda_{m}}\right) . \\
& \left(\left|c_{k_{\mu_{k}-1}}\right|=O\left(e^{\left(-\delta_{2} / 2\right) \lambda_{k}}\right) .\right)
\end{aligned}
$$

But this contradicts with relation (15), and this completes the proof of our theorem.

\section{Conclusion}

In this study we examine a variation of the converse of Fabry Gap theorem.

Polya's result shows that in some sense Fabry's result is the best possible. Perhaps the elementary and direct proof that mentioned above might be of some interest.

To do this, a sequence with a series of new build and reordering the call, using the convergence of three series $f, f^{*}, f^{* *}$ obtain upper and lower bounds. And using the Phragmen-Lindelof theorem and we will achieve the desired result in this paper.

\section{Acknowledgements}

The author gratefully acknowledges the help of Prof. E. Zikkos to improve the original version of the paper.

\section{References}

[1] Abbasi. N., Gorji, M., "On Convergence a Variation of the Converse of Fabry Gap Theorem, " Science Journal of Applied Mathematics and Statistics, 3 (2), (2015), 58-62.

[2] Berenstein, C.A., and Gay Roger, "Complex Analysis and Special Topics in Harmonic Analysis" (New York, Inc: Springer-Verlag), (1995).
[3] Blambert, M. and Parvatham, R., "Ultraconvergence et singualarites pour une classe de series d exponentielles." Universite de Grenoble. Annales de l'Institut Fourier, 29(1), (1979), 239-262.

[4] Blambert, M. and Parvatham, R., "Sur une inegalite fondamentale et les singualarites $d$ une fonction analytique definie par un element LC-dirichletien. "Universite de Grenoble. Annales de l'Institut Fourier, 33(4), (1983), 135-160.

[5] Berland, M., "On the convergenve and singularities of analytic functions defined by E-Dirichletian elements. " Annales des Sciences Mathematiques du Quebec, 22(1),(1998), 1-15.

[6] Boas, R.P. Jr, , "Entire Functions," (New York: Academic Press), (1954).

[7] Erdos, P., "Note on the converse of Fabry's Gap theorem," Trans. Amer. Math. Soc., 57, (1945), 102-104.

[8] Polya, G., "On converse Gap theorems," Trans. Amer. Math. Soc., 52, (1942), 65-71.

[9] Levin, B. Ya., "Distribution of Zeros of Entire Functions," (Providence, R.I.: Amer. Math. Soc.), (1964).

[10] Levin, B. Ya., "Lectures on Entire Functions, " (Providence, R.I.: Amer. Math. Soc.), (1996).

[11] Levinson, N., "Gap and Density Theorems. "American Mathematical Society Colloquium Publications, Vol. 26 (New York: Amer. Math. Soc.), (1940).

[12] Mandelbrojt, S., "Dirichlet Series, Principles and Methods," (Dordrecht: D. Reidel Publishing Co.), (1972), pp. x166.

[13] Valiron, M. G., "Sur les solutions des equations differentielles lineaires d'ordre infni et a coeffcients constants, " Ann.Ecole Norm Trans, 3, 46, (1929), 25-53.

[14] Zikkos, E., "On a theorem of Norman Levinson and a variation of the Fabry Gap theorem," Complex Variables, 50 (4), (2005), 229-255. 